\title{
Neurological manifestations of paranasal disease: problems in prognosis
}

\author{
I. R. LIVINGSTONE \\ M.B., B.Ch., M.R.C.P. \\ W. J. K. Cumming* \\ M.D., F.R.C.P. \\ K. HALL \\ J. B. Foster \\ M.B., B.S., F.R.C.R. \\ M.D., F.R.C.P.
}

Regional Neurological Centre, Newcastle General Hospital, Westgate Road, Newcastle upon Tyne, NE4 6BE

\section{Summary}

Early diagnosis of sphenoidal sinus mucocoeles presenting with visual loss is important as appropriately timed surgical intervention may improve the visual deficit. Two cases of sphenoidal sinus mucocoeles presenting with visual symptoms are reported in whom the diagnosis was only established at a late stage. A third case thought on clinical and radiological grounds to have a sphenoidal sinus mucocoele causing visual symptoms was found to have a frontal astrocytic glioma eroding through the ethmoidal sinus. These case reports stress that the definitive diagnosis in cases of sphenoidal sinus mucocoele is often delayed and that the pre-operative distinction between sphenoethmoidal sinus mucocoeles and other lesions involving the spheno-ethmoidal region may not always be possible.

\section{Introduction}

Mucocoele of the sphenoid or posterior ethmoid paranasal sinuses usually presents with ipsilateral orbital or frontal headache and with involvement of one or more of the cranial nerves which pass through the orbital apex (Everberg, 1955; Nugent, Sprinkle and Bloor, 1970). Visual loss, which is the most common neurological deficit resulting from these lesions, is usually slowly progressive but may be of sudden onset (Everberg, 1955).

The clinical presentations and radiological findings of lesions involving the sphenoido-ethmoidal region in 3 patients with impaired vision are reported, two of whom had sphenoid sinus mucocoeles and one a primary cerebral glioma.

\section{Case reports}

Case 1

This young male initially presented at the age of

\footnotetext{
- Present address: Department of Neurology, Manchester Royal Infirmary, Manchester, England.
}

15 years with a 3-day history of painful loss of vision in the right eye. Clinical examination at that time revealed a sluggish right afferent pupillary response to light with normal appearance of the optic discs. Visual field examination showed a large right central scotoma. A provisional diagnosis of retrobulbar neuritis was made and he was given oral prednisolone for 3 weeks. Within 3 weeks of commencing treatment the pain subsided, and visual acuity returned to normal with complete resolution of the scotoma. $\mathrm{He}$ remained well for 6 months when he again developed painful loss of vision in the right eye with a right central scotoma. He was given a further course of oral steroids again with complete recovery within 14 days at which stage his visual acuity, visual fields and pattern visual evoked responses were normal. A skull radiograph revealed an asymmetrical expansion of the sella more marked on the right than the left with upward displacement of the right anterior clinoid process and impaired definition of the inferior margin of the right optic foramen. Bilateral carotid angiography and air encephaloography were normal. Computerized tomography (CT) of the orbits showed no evidence of optic nerve or orbital pathology. The patient was discharged but was re-admitted 2 months later with a further episode of visual failure in the right eye. Visual acuity was limited to hand movement only and visual field examination again revealed a dense right central scotoma to $20^{\circ}$ with a $20 \mathrm{~mm}$ white target. His visual symptoms and signs improved spontaneously. Pattern visual evoked responses performed at that time revealed that the p100 or major positive component was of normal latency on the left $(120 \mathrm{msec})$ but delayed $(161 \mathrm{msec})$ on the right. Although the visual acuity in the right eye improved it did not return to normal and remained at $6 / 18$ on the right compared to $6 / 6$ on the left. He had two further episodes of painful impaired vision 14 and 16 months respectively after his initial presentation. Both these latter 


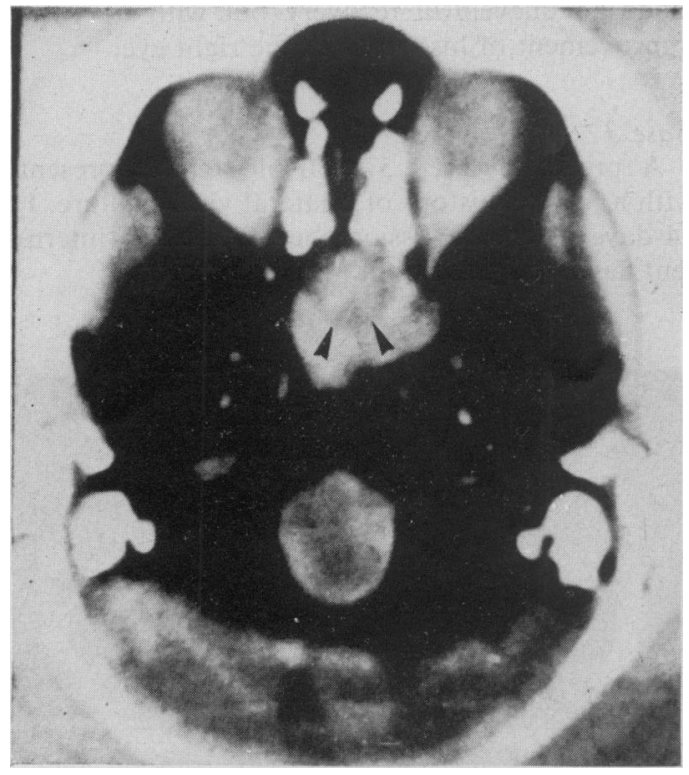

FIG. 1. CT scan (Case 1) showing mucocoele in anterior part of sphenoidal sinus (black arrowheads). (Reproduced by permission of Professor G. du Boulay, Lysholm Department of Radiology, The National Hospital, Queen Square, London.) two episodes of visual failure were preceded by an upper respiratory tract infection. Despite further courses of steroid the best corrected visual acuity in the right eye remained at $6 / 18$. A repeat CT scan revealed features of a large sphenoidal sinus muco-

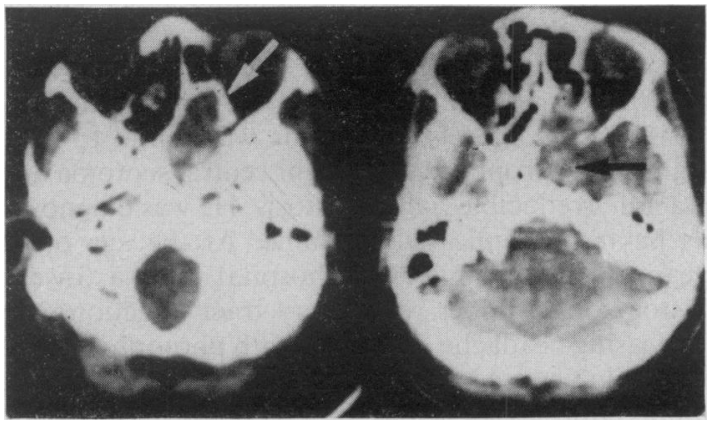

FIG. 2. CT scan (Case 2) showing a right sphenoethmoidal mucocoele expanding the sinus into a right orbit (white arrow) and destroying the right side of pituitary fossa (black arrow).

coele (Fig. 1). Drainage of a $4 \mathrm{~cm}$ sphenoidal mucocoele was carried out through the right ethmoidal approach. The patient made an eventual recovery from surgery and has remained free from further

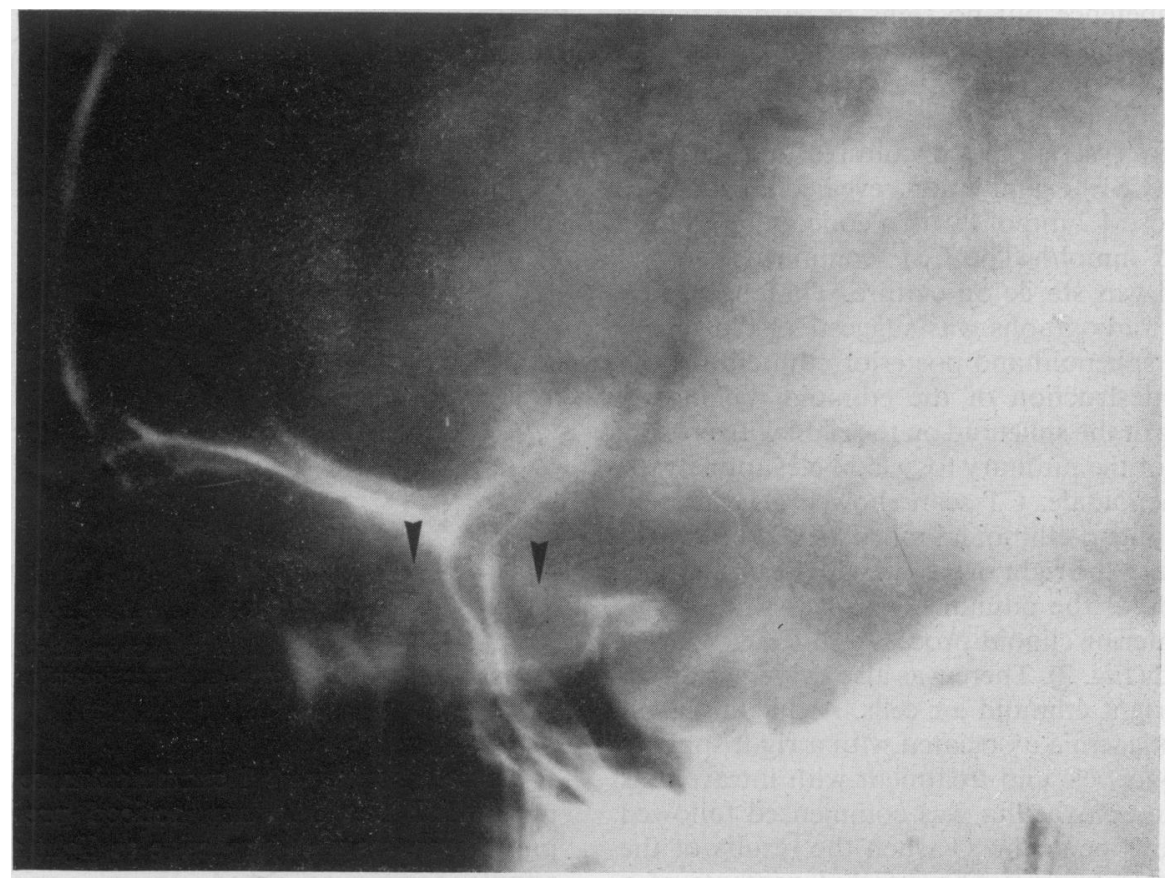

Fig. 3. Lateral skull radiograph (Case 3) showing destruction of planum sphenoidale and cribriform plate (black arrowheads) and evidence of raised intracranial pressure. 
attacks of painful visual loss, although the visual acuity of $6 / 18$ in the right eye has not improved.

\section{Case 2}

This 45-year-old male first presented at the age of 17 years to the ENT department when he underwent a drainage procedure of the ethmoid sinus. He remained well until the age of 28 years when he developed the first of several episodes of painful visual loss in the right eye associated with mild right proptosis. At the age of 34 years he was found to have right optic atrophy, a large right central scotoma and a $2 \mathrm{~mm}$ irreducible right proptosis. He was diagnosed as having complicated migraine. At the age of 44 years he was admitted to hospital with a 3-week history of an upper respiratory tract infection with increasing headache associated with photophobia and increasing nausea and anorexia in the 10 days before admission.

Clinical examination at that time revealed a pyrexia of $38^{\circ} \mathrm{C}$. He had bilaterally tender and swollen parotid and lachrymal glands. He was mildly photophobic and had terminal neck stiffness. There was a divergent non-paralytic strabismus with a $2 \mathrm{~mm}$ proptosis of the right eye. He had right optic atrophy and a right central scotoma. Visual acuity was $6 / 36$ in the right eye, and 6/6 in the left eye. The remainder of the neurological examination was normal. General examination revealed mild aortic valve incompetence but no signs of cardiac failure or bacterial endocarditis. Investigation showed: haemoglobin of $14.1 \mathrm{~g} / \mathrm{dl}$; white cell count $24 \times 10^{9} / 1$ predominantly polymorphs. Pneumococcus was grown on four serial blood cultures and cerebrospinal fluid (CSF) examination revealed a protein of $0.6 \mathrm{~g} / \mathrm{l}$, glucose $4.2 \mathrm{mmol} / \mathrm{l}$ with a concomitant blood sugar of $6.5 \mathrm{mmol} / \mathrm{l}$. The CSF contained 11 polymorphs and was sterile on culture. The appearance of the skull radiographs was suggestive of a mucocoele in the sphenoid and posterior ethmoid region. There was destruction of the ethmoid region and greater wing of the sphenoid on the right with erosion of the floor of the pituitary fossa and elevation of the planum sphenoidale. CT scan showed a large nonenhancing spheno-ethmoidal mucocoele on the right expanding into the right orbit but with destruction of the right side of the pituitary fossa extending to involve the anterior clinoid process and lesser wing of the sphenoid (Fig. 2). There was also evident destruction of the right ethmoid air cells. A diagnosis was made of septicaemia associated with a right sphenoethmoidal pyocoele and treatment with intravenous ampicillin and cloxacillin was commenced followed by intravenous penicillin $G$ when the results of the culture became known. The infected contents of a $6 \mathrm{~cm}$ right spheno-ethmoidal mucocoele were then drained through the ethmoidal approach. The patient made an uneventful recovery but with no further improvement of his vision in the right eye.

\section{Case 3}

A previously well 37-year-old female presented with a 3-week history of bilateral visual failure. For 14 days before admission she experienced intermittent flashing lights in both fields of vision.

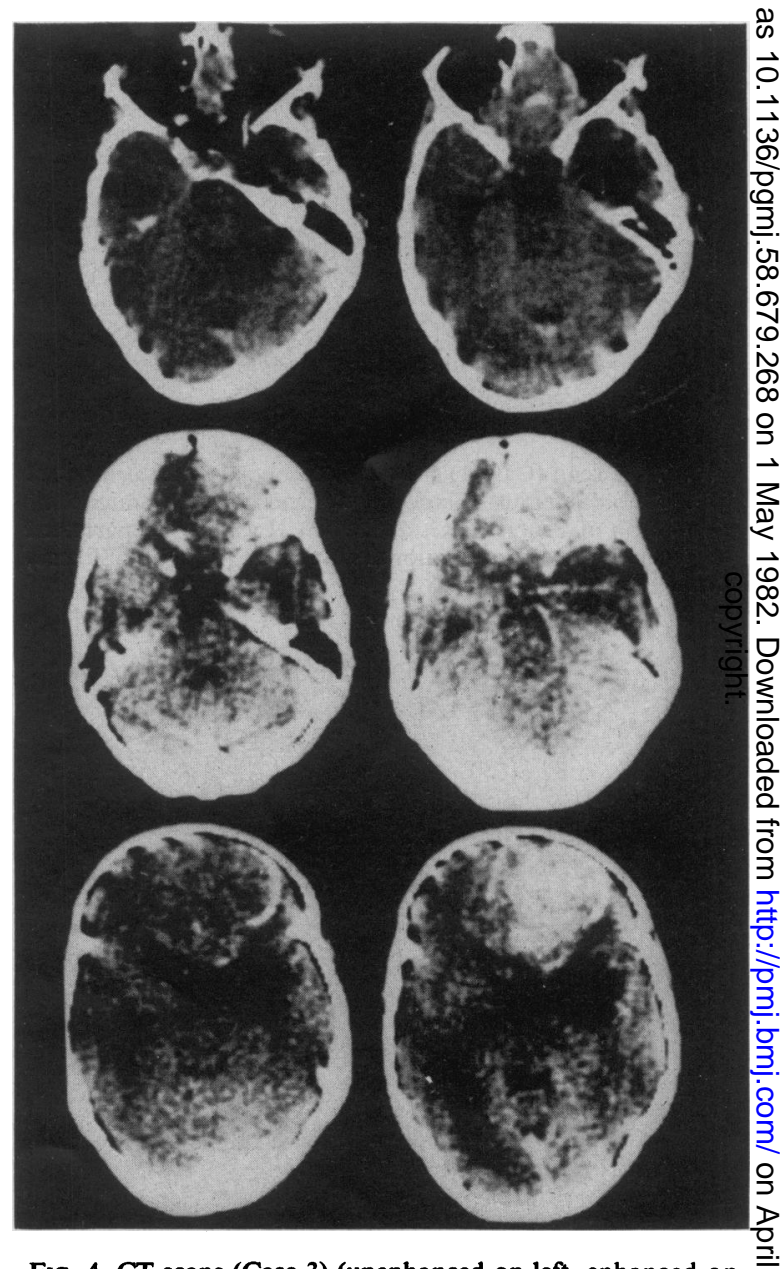

Fig. 4. CT scans (Case 3) (unenhanced on left, enhanced on $\bar{N}$ right) showing a large enhancing mass in the right frontal lobe $\sigma$ extending into the ethmoidal and anterior sphenoidal regions.

Clinical neurological examination revealed anosmia which she had not noticed previously. The pupils were normally reactive to light and bilateral papilloedema was noted. Visual acuity was 6/18 right and 6/12 left, and the yisual fields showed bilateral central scotomata. Ocular movements were 
normal and there was a $2 \mathrm{~mm}$ non-reducible axial proptosis of the right eye. The remainder of the neurological examination was normal as was the general clinical examination.

The skull radiograph revealed erosion of the medial wall of the right orbit with loss of the planum sphenoidale and the cribriform plate. There was also evidence of raised intracranial pressure with loss of the lamina dura of the pituitary fossa (Fig. 3). A CT scan (Fig. 4) showed a large mass arising from the ethmoidal and anterior sphenoidal region extending upwards into the frontal lobe on the right. The lesion was of the same density as the surrounding brain and the edge of the lesion showed curvilinear calcification. There was patchy enhancement following the administration of intravenous contrast. This was considered to be a sphenoido-ethmoidal mucocoele with intracranial extension on the right. An attempt was made to drain the lesion through an anterior ethmoidal approach but at operation the lesion was noted to be a tumour of firm consistency and a biopsy revealed grade 3 astrocytoma. A right carotid angiogram was performed on the following day and this showed slight narrowing of the right anterior cerebral artery with pooling of the contrast medium in the capillary phase. A partial right frontal lobectomy was performed through a right frontal craniotomy and the intracranial tumour was noted to have eroded through the dura into the right ethmoid sinus. Histology of both the intradural and extradural mass revealed a grade 3 astrocytic glioma. This patient received a postoperative course of external radiotherapy with no subsequent improvement in her visual function.

\section{Discussion}

The first detailed description of sphenoidal mucocoeles was by Berg in 1889. Since then various clinical manifestations of sphenoidal sinus mucocoeles have been described (Bilchick, 1940; Everberg, 1955; Goodwin and Glaser, 1978; Hakuba et al., 1975; Herman and Hall, 1945; Krueger, McFarland and Ommaya, 1965; Lundgren and Olin, 1961; Nugent et al., 1970; O'Shea, 1932; Pincus and Daroff, 1964; Reinecke and Montgomery, 1964). A history of previous ENT surgery for paranasal sinus disease is commonplace (Nugent et al., 1970) and often ignored in history taking because of the long interval between the original ENT surgery and the presentation with neurological symptoms.

Visual involvement is a common neurological manifestation of sphenoidal sinus mucocoeles and this may take the form of visual loss which is either steadily progressive or punctuated by relapses and partial subsequent improvements mimicking retrobulbar neuritis or acute demyelinating optic neuritis (Bilchick, 1940; Everberg, 1955; Krueger et al.,
1965). Our 3 cases all presented with visual loss, the first case being initially diagnosed as retrobulbar neuritis. Case 2 was originally assessed as having complicated migraine and in this regard it is of interest to note that several reports have stressed the importance of excluding sphenoidal sinus mucocoeles in patients with complicated migraine especially the ophthalmoplegic migraine syndrome (Herman and Hall, 1945; Pincus and Daroff, 1964). Case 3 presented with a short history of bilateral visual loss and although visual symptoms are usually unilateral in sphenoidal sinus disease, bilateral visual involvement has been reported by Nugent $e t$ al. (1970) in 7 of 41 patients with sphenoidal sinus mucocoeles presenting with visual loss.

Non-pulsatile proptosis is similarly well described with sphenoidal sinus mucocoeles (Nugent et al., 1970) but pulsatile exophthalmos by contrast is notably rare (O'Shea, 1932). Anosmia, which was a feature in case 3, has been recorded in 9 out of 63 presented with sphenoidal sinus mucocoeles (Nugent et al., 1970).

There may be some clinical grounds for distinguishing sphenoidal from ethmoidal sinus mucocoeles (Petit-Dutailles, Thiebaut and Fischgold, $1950)$ as the sphenoidal sinus mucocoele usually has a longer history and visual loss tends to occur later. By contrast the ethmoid sinus mucocoele syndrome is usually associated with optic disc swelling and less frequently with anosmia and exophthalmus. That the clinical features of case 3 fall into this latter diagnostic group is confirmed by the radiological appearances which show the ethmoidal region to be the major site of extradural involvement.

The optimal surgical approach to sphenoidal sinus mucocoeles is via the nasal or oral route. The risks of craniotomy are well exemplified by the report of 4 deaths attributable to such an approach (Lundgren and Olin, 1961), risks that are especially great in the presence of infection. Specific preoperative radiological diagnosis is thus very important in determining the surgical approach to lesions of the sphenoid and posterior ethmoidal region. Posterior mucocoeles were not an uncommon finding in the course of surgery for parasellar tumours in the days before the specific radiological features of sphenoidal and posterior ethmoidal sinus mucocoeles were defined (Petit-Dutailles et al., 1950). More accurate preoperative diagnosis has resulted from the description of the diverse radiological features of sphenoid sinus mucocoeles (Dubois et al., 1977; Hakuba et al., 1975; Hesselink et al., 1979; Minagi, Margolis and Newton, 1972; Takahashi, Jungu and Nakayama, 1973).

Although sphenoidal mucocoeles may rarely present as an intrasellar mass (Osborn, Johnson and Roberts, 1979; Simms, Brown and French, 1970) 
the distinction between lesions arising from the sphenoid sinus and pituitary tumours usually rests on demonstrating the absence of sellar enlargement in sphenoid sinus lesions (Hakuba et al., 1975; Robertson et al., 1976), despite destruction of the floor of the sella. Several reports have stressed the importance of detailed tomography on several planes in defining the nature of lesions involving the ethmoid and sphenoid sinuses (Dubois et al., 1977; Minagi et al., 1972; Robertson et al., 1976; Takahashi et al., 1973). Opacification of the sinus and expansion and thinning of the sinus walls has been reported as a consistent finding with sphenoid sinus mucocoeles in one series of 14 cases (Robertson et al., 1976). Axial CT scanning alone is not always sufficient for adequate evaluation of paranasal sinus mucocoeles (Hesselink et al., 1979) and needs to be supplemented with either coronal CT scanning or plain tomography. In a review of the CT scan appearance of 18 paranasal sinus mucocoeles (Hesselink et al., 1979), rim enhancement was noted to occur only in the presence of acute infection as, in the absence of infection, the mucocoeles are usually of the same density as the brain.

The erosion through the dura by a frontal astrocytic glioma into the ethmoidal in case 3 is unusual. Transdural extension of a glioma is a rare event as the dura is resistant to penetration. Infiltration of the dura by glioblastomas and anaplastic astrocytomas is usually confined to the inner dural layer (Russell and Rubinstein, 1971), Cases of transdural extension are, however, described. Rubinstein (1967) described infiltration of the sagittal venous sinus by an anaplastic astrocytoma. Russell and Rubinstein (1971) describe a case of a 7-year-old girl with a superficial temporal glioblastoma with erosion through the great wing of the sphenoid and sellar turcica. Two further reports have described spontaneous transdural extension of temporal lobe glioblastomas with invasion of the temporal bone (Nager, 1967; Sanerkin, 1962).

The clinical features of our 3 reported cases suggest that early diagnosis in cases of the sphenoidal sinus mucocoele syndrome presenting with visual symptoms is still difficult in many instances and that several radiological examinations may be required for diagnosis. Furthermore, the preoperative differentiation of mucocoeles from other lesions in the region of the ethmoid and sphenoidal sinuses in patients presenting with the sphenoidal or posterior ethmoidal syndrome may not always be possible.

\section{Acknowledgment}

We wish to thank Dr P. Hudgson for permission to report Case 1.

\section{References}

BERG, J. (1889) Bidrag till kannendomen om sjukdomarna: nasans samt till laran om cerebro-spinal vatskas flytning ur nasan. 2. Hydrops sinus sphenoidalis. Nordisk Medicinhistorisk Aarsbok, 21, 5.

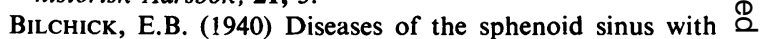
report of a case of cyst of the sphenoid sinus. Archives of $C$ Otolaryngology, 32, 1031.

Dubois, P.J., Schultz, J.C., Perrin, R.L. \& Dastur, K.J. G (1977) Tomography in expansile lesions of the nasal and paranasal sinuses. Radiology, 125, 149.

EVERBERG, G. (1955) Retrobulbar neuritis due to cyst of sphenoid sinus. Acta oto-laryngologica (Stockholm), 45, 492.

Goodwin, J.A. \& Glaser, J.S. (1978) Chiasmal syndrome in sphenoid sinus mucocele. Annals of Neurology, 4, 440.

Hakuba, A., Katsuyama, J., Matsuoka, Y., Hong Shim, J. \& Nishimura, S. (1975) Sphenoid sinus mucocoeles. Report of two cases. Journal of Neurosurgery, 43, 368.

Herman, K. \& Hall, I.S. (1945) Sphenoidal mucocoele as a cause of the 'opthalmoplegic migraine syndrome'. Transactions of the Ophthalmological Society of the United Kingdom, 64, 154.

Hesselink, J.R., Weber, A.L., New, P.F., Davis, K.R., Roberson, G.H. \& TAVeras, J.M. (1979) Evaluation of mucocoeles of the paranasal sinuses with computed tomography. Radiology, 133, 397.

Krueger, T.P., McFarland, J. \& OmmayA, A.K. (1965) Pyocoele of the sphenoid sinus. Journal of Neurosurgery. 22, 616 .

Lundgren, A. \& Olin, T. (1961) Mucopyocoele of the sphenoidal sinus or posterior ethmoidal cells with special reference to the apex orbitae syndrome. Acta oto-laryngologica (Stockholm), 53, 61.

Minagi, H., Margolis, T. \& Newton, T.H. (1972) Tomoe graphy in the diagnosis of sphenoid sinus mucocele American Journal of Roentgenology, 115, 587.

NAGER, G.T. (1967) Gliomas involving the temporal bone? Clinical and pathological aspects. Laryngoscope (St Louis), 77, 454.

Nugent, G.R., SPrinkle, P. \& Bloor, B.M. (1970) Sphenoid sinus mucocoeles. Journal of Neurosurgery, 32, 443.

Osborn, A.G., Johnson, L. \& Roberts, T.S. (1979) Sphenoidal mucocoeles with intracranial extension. Journal of Computer Assisted Tomography, 3, 335.

O'SheA, H.V. (1932) Pulsating exophthalmos due to sphenoidal sinus mucocoele. Lancet, i, 1253.

Petit-Dutailles, D., Thiebaut, F. \& Fischgold, H. (1950) Contribution à l'étude des compressions intracraniennes des nerfs optiques par les abcés ou les mucocoeles extradurales d'origine sphénoéthmoidale. Revue Neurologique (Paris), 83, 325.

Pincus, J.G. \& Daroff, R.B. (1964) Sphenoid sinus mucoceles. A curable cause of the ophthalmoplegic migraine syndrome. Journal of the American Medical Association, $187,459$.

Reinecke, R.D. \& Montgomery, W.W.M. (1964) Oculomotor nerve palsy assuciated with mucocele of the sphenoid sinus. Archives of Ophthalmology, 71, 50.

Robertson, G.H., Patterson, A.K., El Deeb, M., Maisel, R.H. \& BONE, R.C. (1976) Sphenoethmoidal mucocele: Radiographic diagnosis. American Journal of Roentgeno$\log y, 127,595$.

RubinsteIN, L.J. (1967) Development of extracranial metastases from a malignant astrocytoma. Journal of Neurosurgery, 26, 542.

Russell, D.S., Rubinstein, L.J. (1971) Pathology of tumours of the nervous system. 3rd edn. Edward Arnold, London.

SANERKIN, N.G. (1962) Transdural spread of glioblastoma multiforme. Journal of Pathology, 84, 228. 
Simms, N.M., Brown, W.E. \& French, L.A. (1970) Mucocoele of the sphenoid sinus presenting as an intrasellar mass. Journal of Neurosurgery, 32, 710.
Takahashi, M., Jingu, K. \& Nakayama, T. (1973) Roentgenologic appearances of sphenoethmoidal mucocoele. Neuroradiology, 6, 45. 